\title{
PARP inhibitor olaparib is safe and effective in patients with BRCA1 and BRCA2 mutations
}

W

omen who harbor BRCA1

or BRCA2 mutations have a

high risk of developing breast and ovarian cancer. The BRCA 1 and $B R C A 2$ gene products are involved in a specialized form of DNA repair, known as homologous recombination. If the wild type BRCA allele in a tumor cell is lost, the repair mechanism is compromised and the resulting genetic instability can cause tumor development. Poly (ADP ribose) polymerase (PARP) is involved in repairing single-strand DNA breaks, and inhibition of PARP results in single-strand breaks that accumulate to form doublestrand breaks. These errors are repaired by components of the homologous recombination repair pathway, which includes BRCA1 and BRCA2. However, tumors that cannot repair their DNA owing to compromised BRCA function are highly sensitive to PARP inhibition. This finding provides the concept of synthetic lethality, whereby a lethal synergy occurs between two non-lethal events-in this case inhibition of PARP induces a genetic lesion that is lethal in patients who lack a functional DNA repair pathway owing to loss of BRCA function.

Previous research by Alan Ashworth's group in cell line models showed that when DNA repair is defective owing to loss of BRCA function, this defect was lethal when combined with induced loss of PARP, but only when cells lacked functional BRCA. Thus, treatment with PARP inhibitors could be particularly beneficial in women with $B R C A$-mutated breast and ovarian cancers. Andrew Tutt, from Alan Ashworth's group together with other international investigators, set out to show whether PARP was selectively lethal for tumors with loss of function of $B R C A 1$ and BRCA2.
Olaparib is a novel, orally active PARP inhibitor that induces synthetic lethality in homozygous BRCA-mutated cells. A phase I study revealed that olaparib was safe and well tolerated as a single agent in $B R C A$ mutation carriers. Therefore, two proof-of-concept phase II studies-one lead by Andrew Tutt and the other by William Audeh-were carried out to test olaparib safety and efficacy in patients with advanced or recurrent breast or ovarian cancer who had BRCA1 or BRCA2 mutations. "Targeting loss of BRCA1 or BRCA2 function with a PARP inhibitor can lead to a meaningful anticancer activity in patients who have had or become resistant to other forms of standard chemotherapy" explains Tutt.

In the first phase II trial by Tutt and coauthors, women with advanced breast cancer and $B R C A 1$ or $B R C A 2$ mutations who had received at least one chemotherapy regimen were enrolled in two sequential cohorts from 16 centers. In the first cohort, patients received $400 \mathrm{mg}$ olaparib twice daily, and in the second cohort they received $100 \mathrm{mg}$ olaparib twice daily. The primary end point was objective response rate (ORR) and safety and tolerability were also assessed. The ORR was higher in the first cohort than the second (41\% versus $22 \%$ ) and toxic effects - fatigue, nausea and vomitingwere mainly low grade and manageable. The median progression-free survival was 5.7 months for the high-dose cohort and 3.8 months for the low-dose cohort.

In the second phase II trial led by William Audeh, the efficacy and safety of olaparib was tested at the $100 \mathrm{mg}$ and $400 \mathrm{mg}$ doses in women with recurrent ovarian cancer. In both phase II studies, as the frequency of early progression was higher in the low-dose group the studies were amended to allow patients to receive the higher dose if disease progression occurred. The ORR (primary end point of the trial) was 33\% for the high-dose cohort and $13 \%$ for the low-dose cohort. Median progression-free survival was 5.8 months for the high-dose group and 1.9 months for the low-dose group. The clinical benefit rate was higher in women receiving the $400 \mathrm{mg}$ dose. The most frequent adverse effects were nausea and fatigue, but in general these were mild in intensity. As Audeh observes "these patients were heavily pre-treated and some have had prolonged partial and complete responses".

The results from both studies show that the underlying pathways that are aberrant may be more important than the organ of origin when selecting therapy. "Currently, the presence of mutations in BRCA1 or $B R C A 2$ does not inform systemic therapy recommendations for women with breast cancer, but the results of this and subsequent studies might change established practice" concludes Tutt. Audeh also notes "The implications of our trial suggest that a broader group of patients may benefit from PARP inhibitor therapy". Andrew Tutt and his colleagues are now developing PARP inhibitors to test in clinical trials as single agents and in combination with standard therapies in a wider range of sporadic and $B R C A 1$ BRCA 2 associated breast, ovarian and other solid tumors.

\section{Lisa Hutchinson}
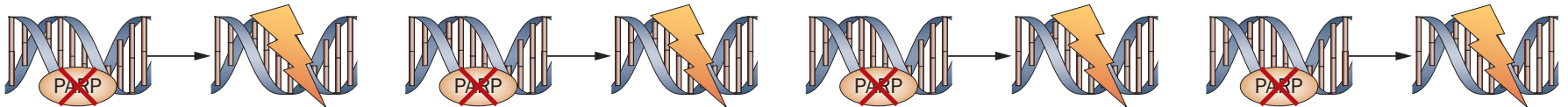